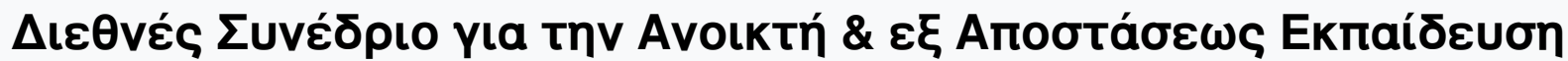

Tóp. 6, Ap. 2B (2011)

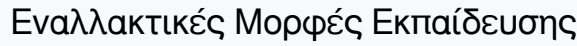

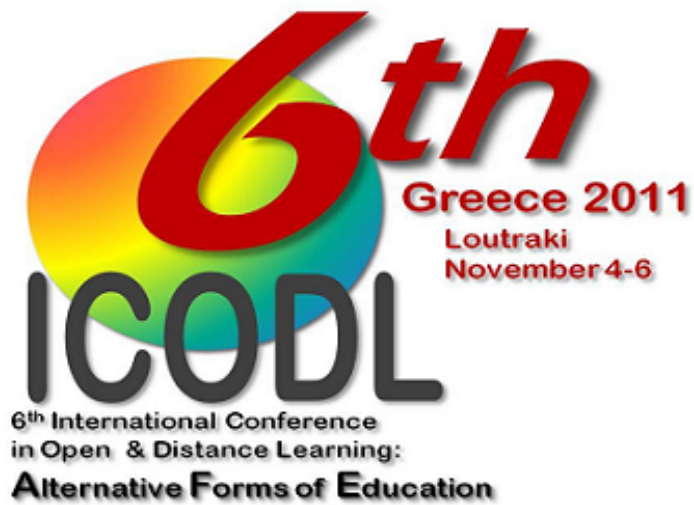

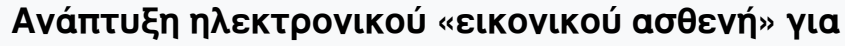

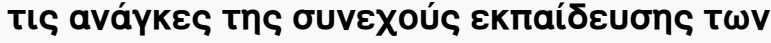
oठovtıátpwV

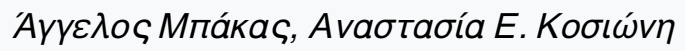

doi: $10.12681 /$ icodl. 666

TOMOE B

PART / MEPOE B 


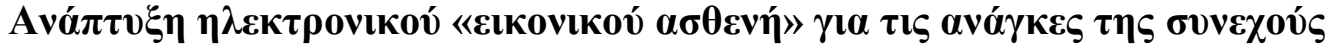

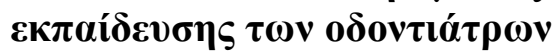

\section{The development of a "virtual patient" case for the continuing professional education of dentists}

\author{
А' $\gamma \gamma \varepsilon \lambda о \varsigma$ М $\pi \alpha ́ \kappa \alpha \varsigma$

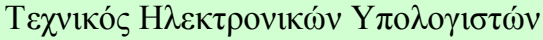

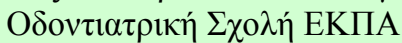 \\ abakas@dent.uoa.gr
}

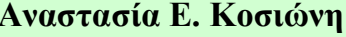

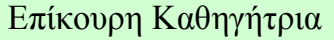

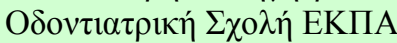 \\ akossion@dent.uoa.gr
}

\begin{abstract}
The aim of this study was to describe the design and development of a "virtual patient" case for the continuing professional education of graduate dentists in Greece. The "virtual patients" are interactive computer simulations of various clinical conditions, applied in all levels of healthcare education, providing training and assessment. They provide a reliable and safe educational environment in which to practice diagnostic skills and make clinical decisions. Two software programmes were applied VUE and OpenLabyrinth. The scenario was related to the management of an older medically compromised dental patient and was developed by an expert in Geriatric Dentistry with special knowledge in open and distance learning and adult learning. The application was implemented by a computer programmer. Clinical photographs, radiographs and avatars were incorporated in the final case. The scenario was further evaluated by dental academic experts and pretested in 10 dental professionals to diagnose any technical problems. The dentist could choose online from a variety of different clinical decisions the one he/ she preferred. For each choice he/ she received immediate feedback from an "expert". A summative self-assessment at the end of the case presented the decisions that should have been avoided and the time spent in each node (internet page). An initial evaluation was added to investigate the sociodemographic data of the learners and their knowledge and attitudes towards treating the elderly. A final evaluation including both close-ended and open-ended questions evaluated all aspects of the application and the learning outcomes. The "virtual patients" are an important innovation which enhances self-directed learning at the time, place and pace of each individual learner and can be a valuable alternative educational method in the continuing education of the health professionals.
\end{abstract}

Key-words: virtual patient, continuing professional development, health professions

\section{Пєрі́ $\eta \psi \eta$}

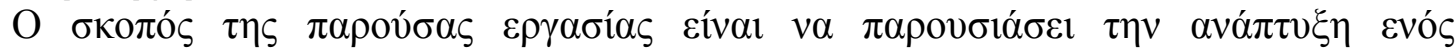

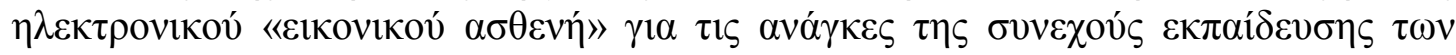

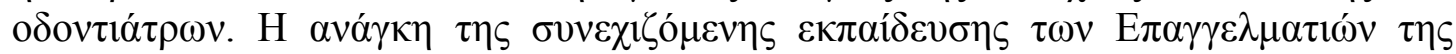

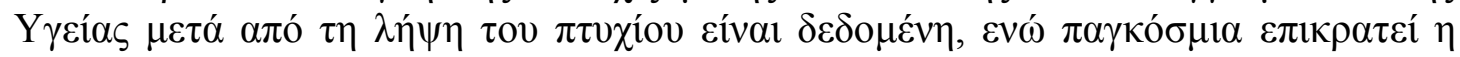

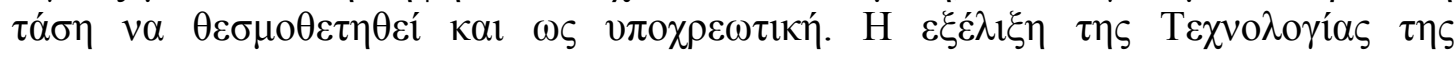

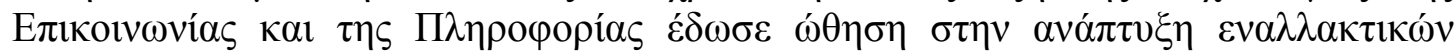




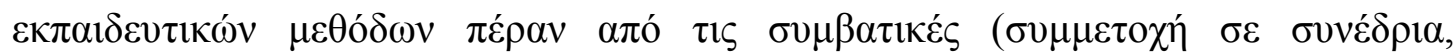

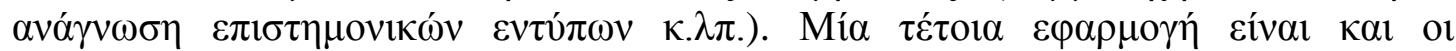

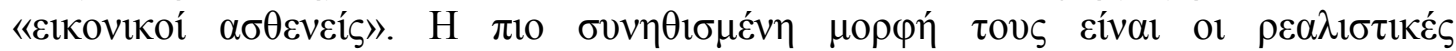

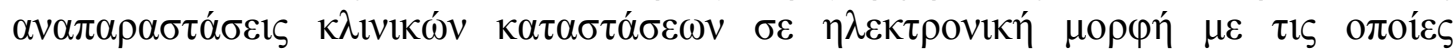

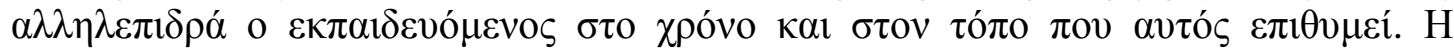

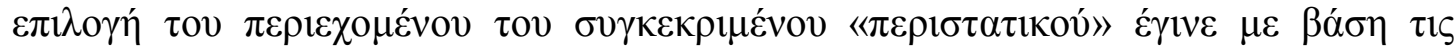

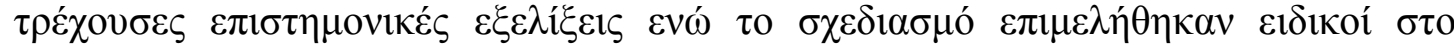

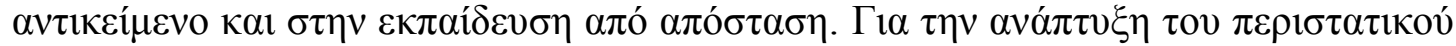

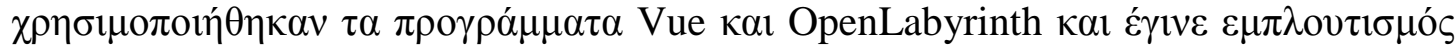

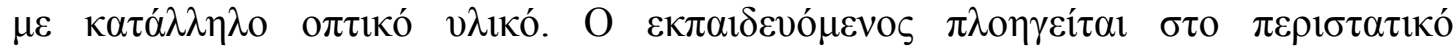

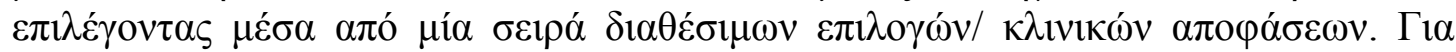

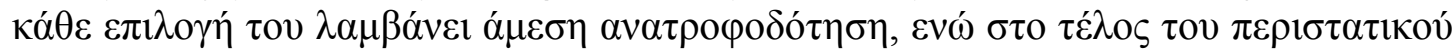

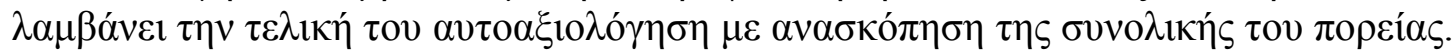

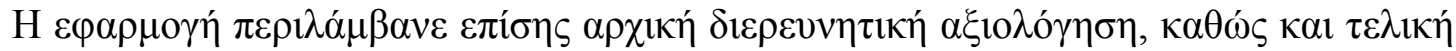

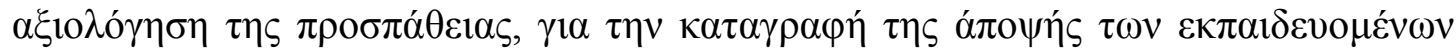

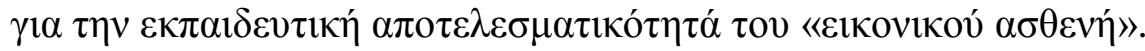

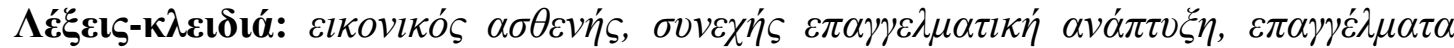
vүeías

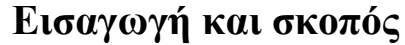

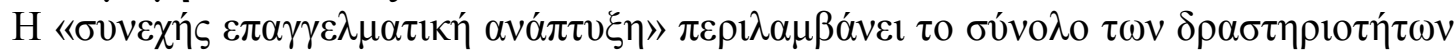

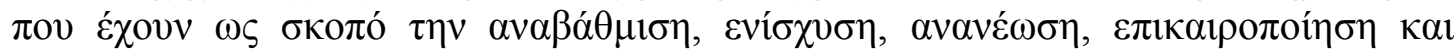

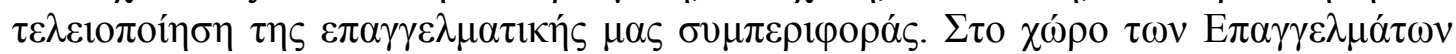

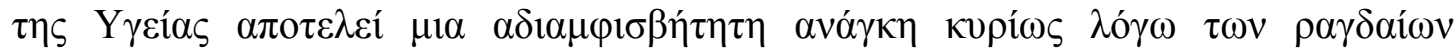

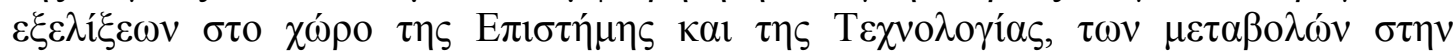

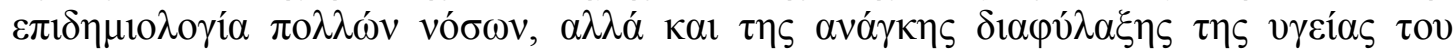

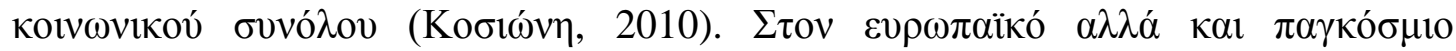

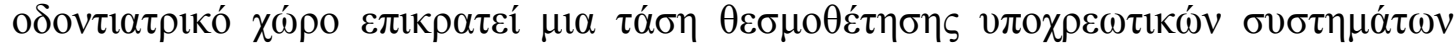

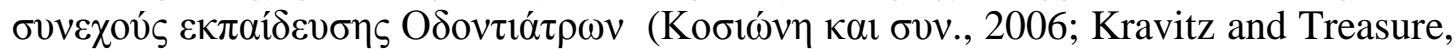

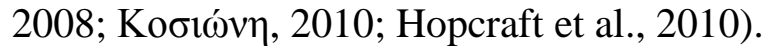

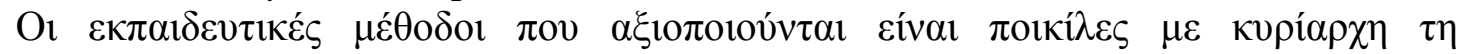

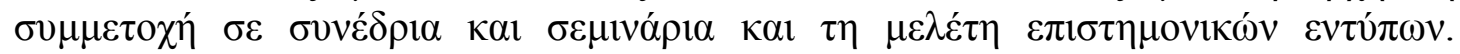

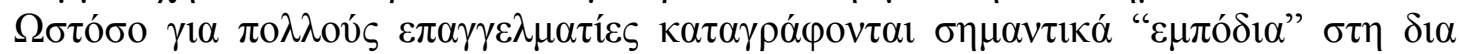

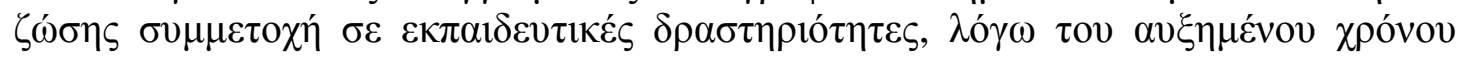

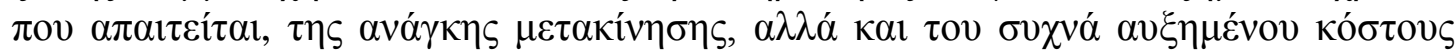

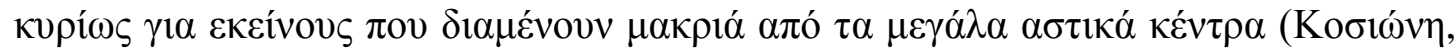

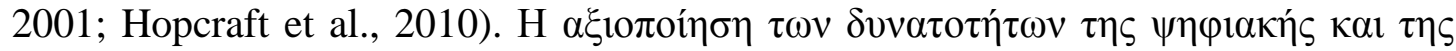

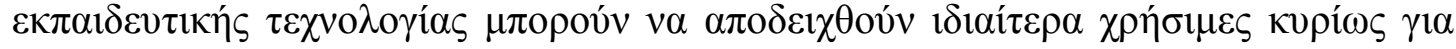

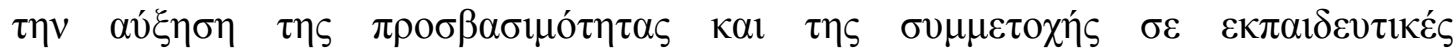

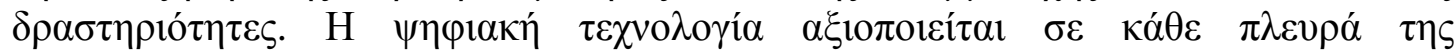

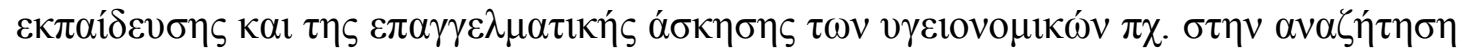

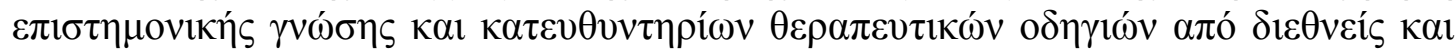

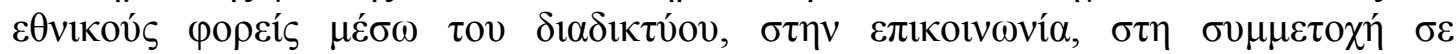

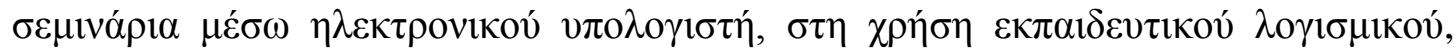

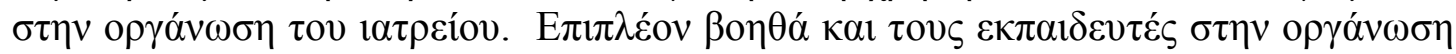

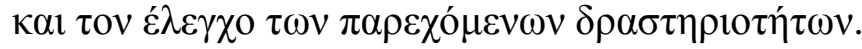




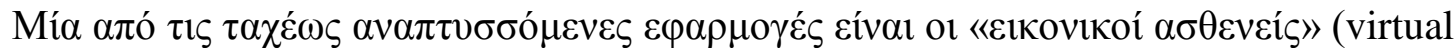

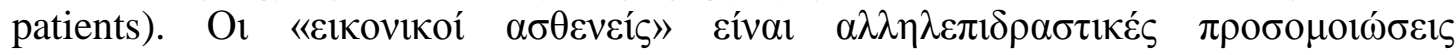

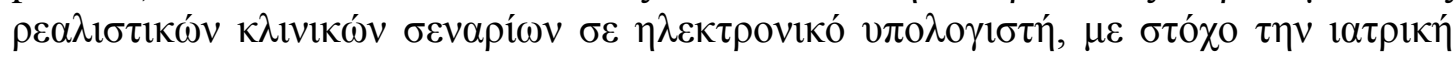

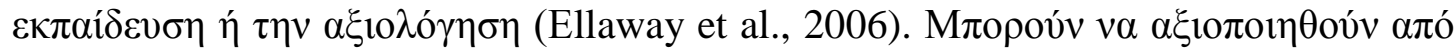

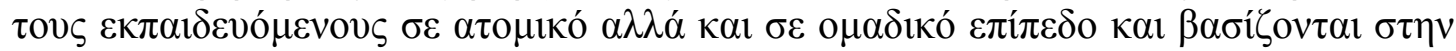

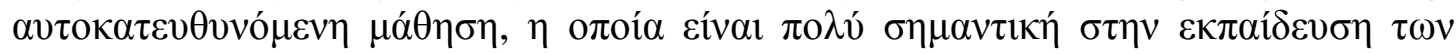

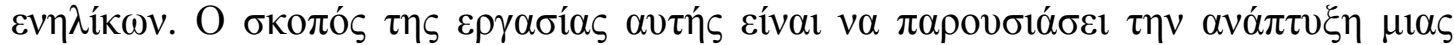

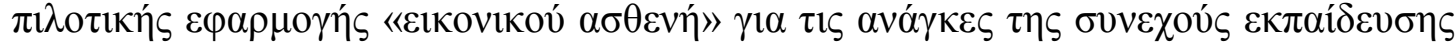

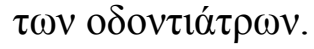

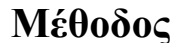

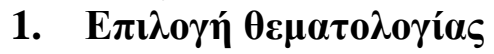

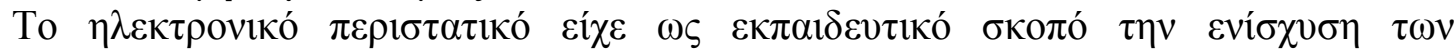

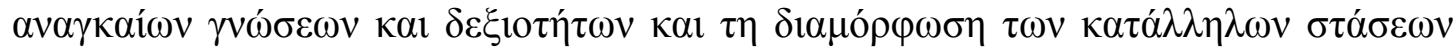

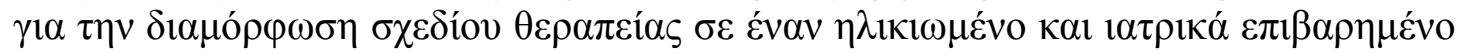

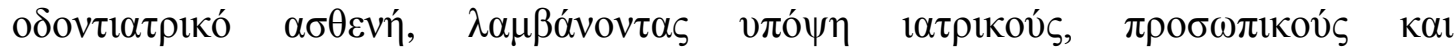

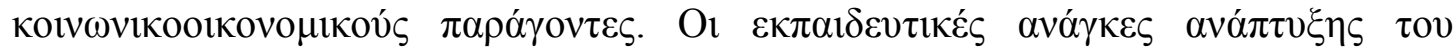

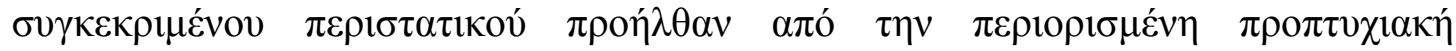

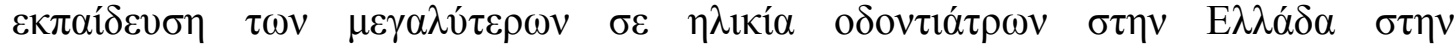

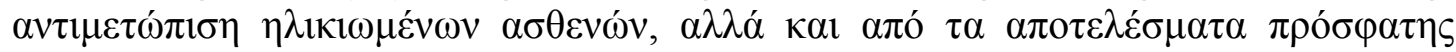

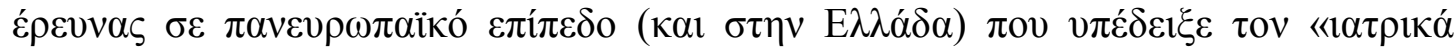

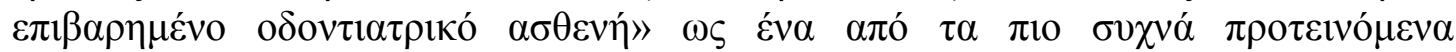

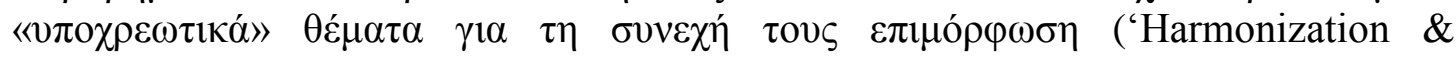
Standardization of European Dental Schools' Programs of Continuing Professional Development for Graduate Dentists'/ DentCPD , ref: 509961-LLP-1-2010-1-UKERASMUS-EMHE).

\section{Avó $\pi \tau v \xi \eta \eta \pi \varepsilon \rho \varepsilon \chi 0 \mu \varepsilon ́ v o v ~ \mu \alpha \theta \eta ́ n \alpha \tau 0 \varsigma$}

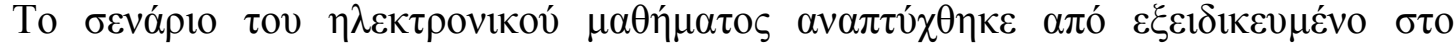

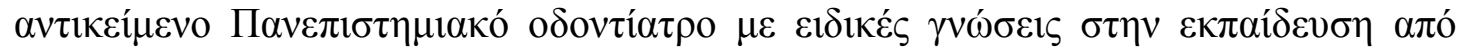

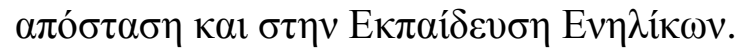

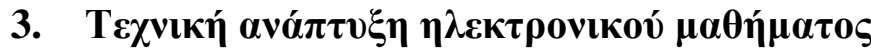

Гı

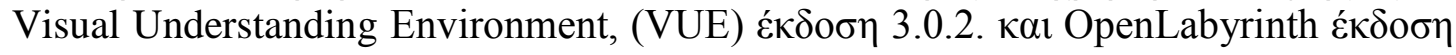

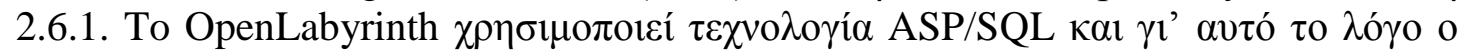

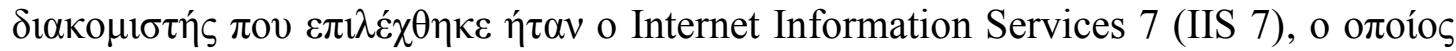

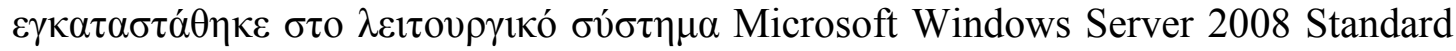

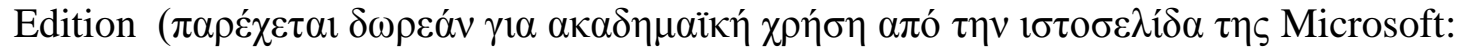

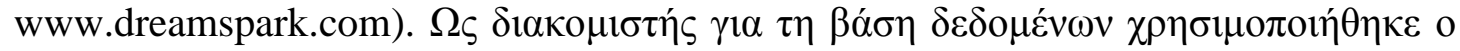

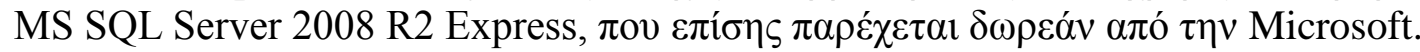

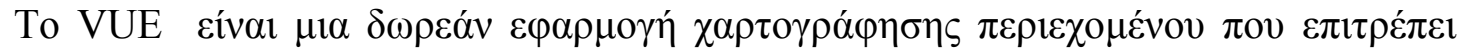

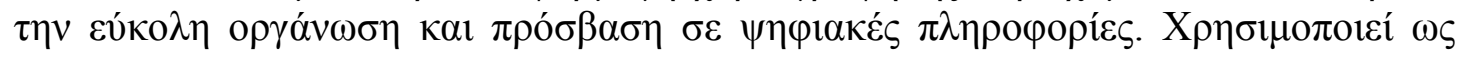

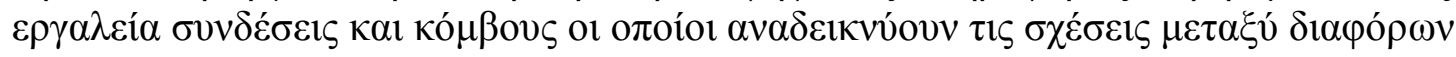
$1 \delta \varepsilon \omega ́ v, \quad \alpha \rho \chi \omega ́ v \quad \kappa \alpha 1 \quad \delta \varepsilon \delta 0 \mu \varepsilon ́ v \omega v$ (http://vue.tufts.edu/about/index.cfm). To

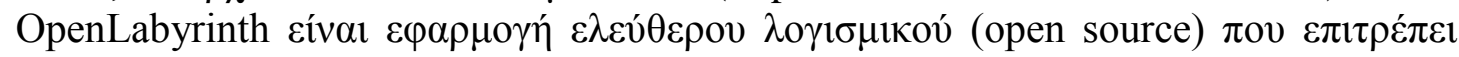

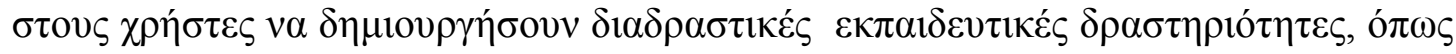

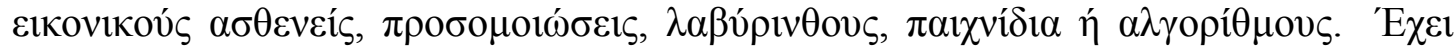




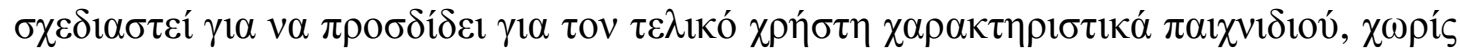

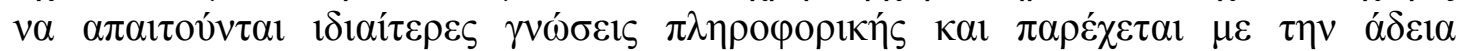

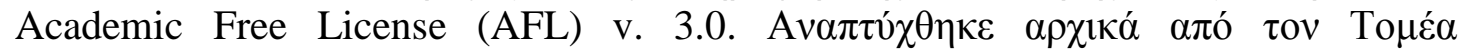

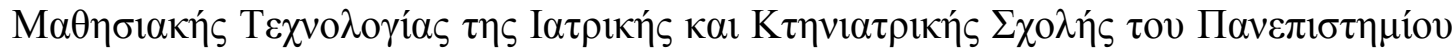

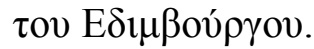

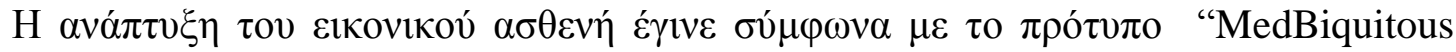

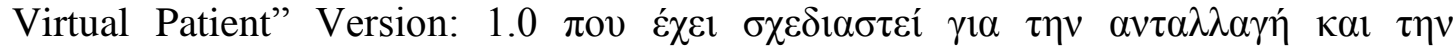

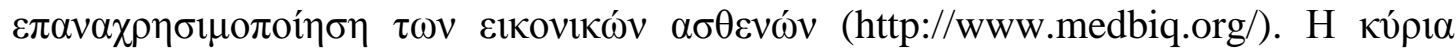

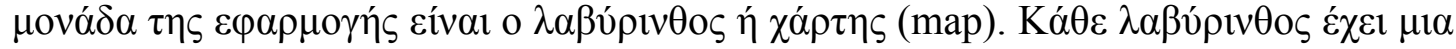

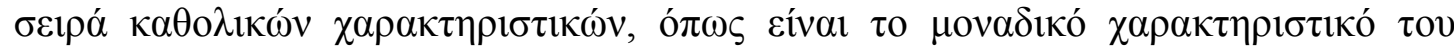

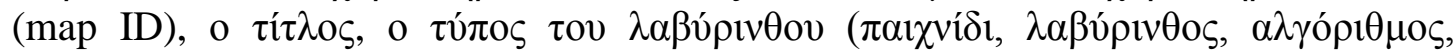

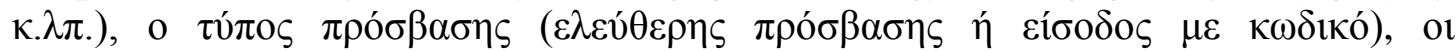

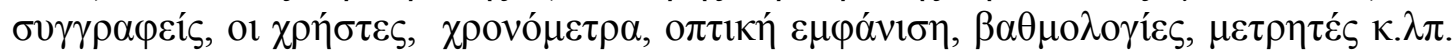

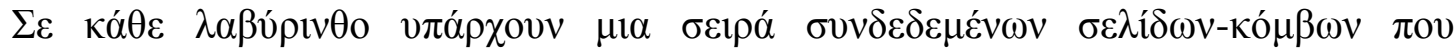

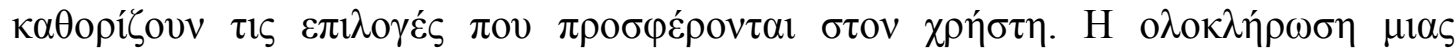

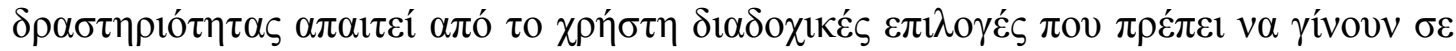

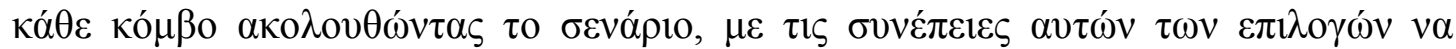

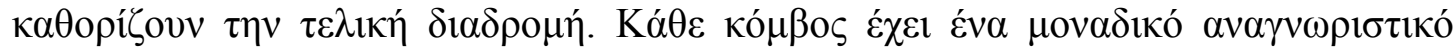

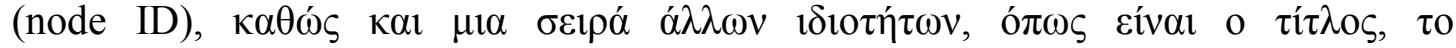

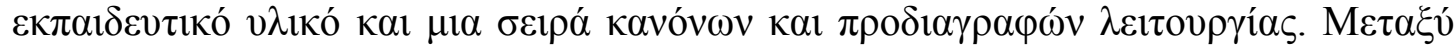

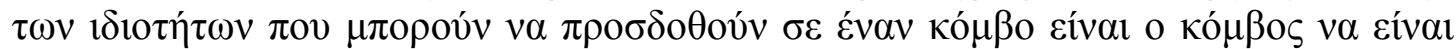

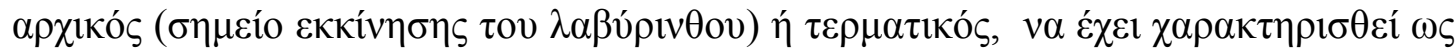

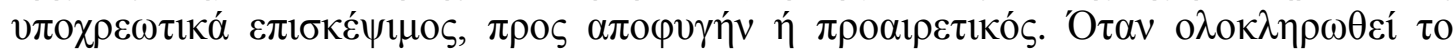

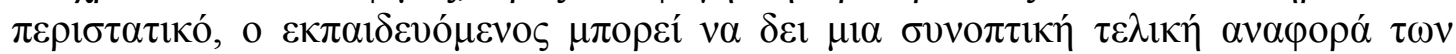

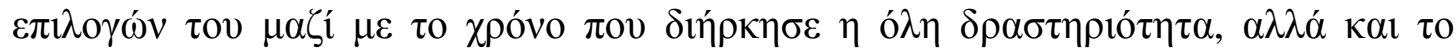

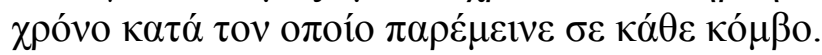

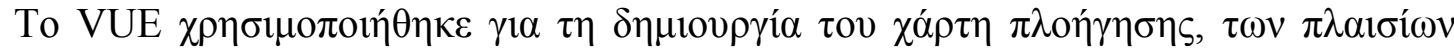

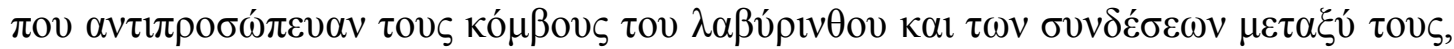

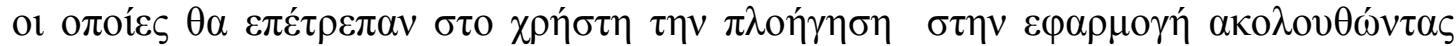

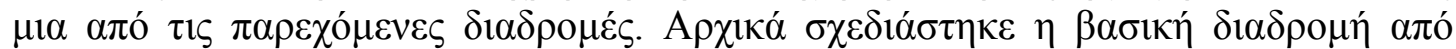

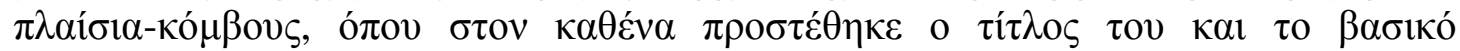

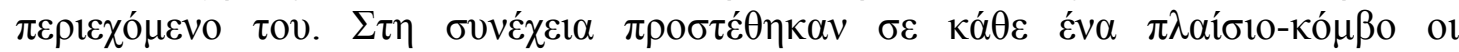

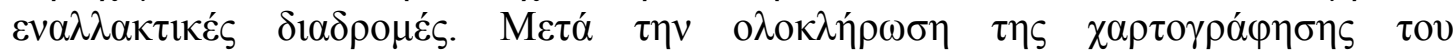

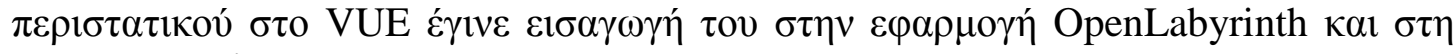

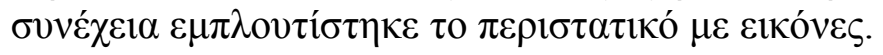

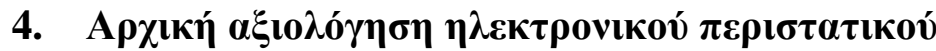

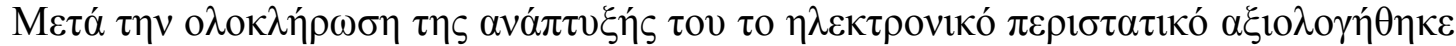

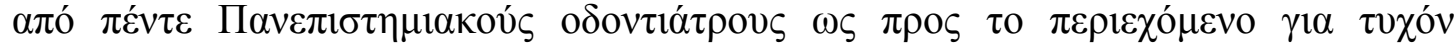

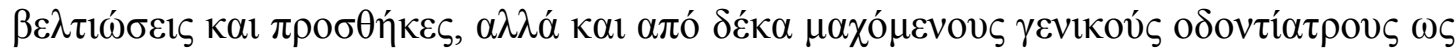

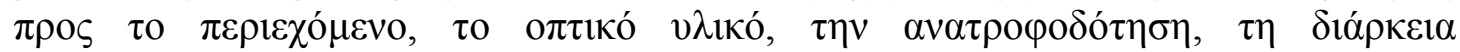

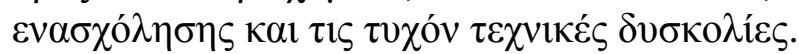

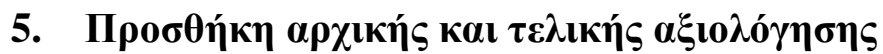

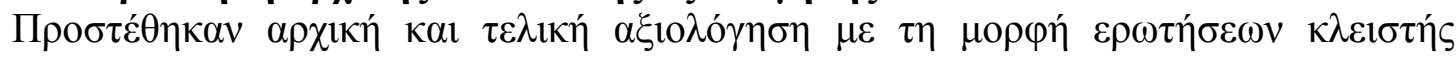

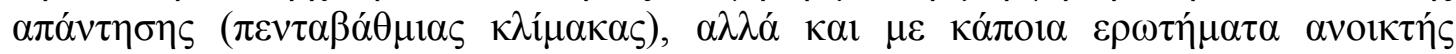
$\alpha \pi \alpha ́ v \tau \eta \sigma \eta s$. 


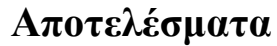

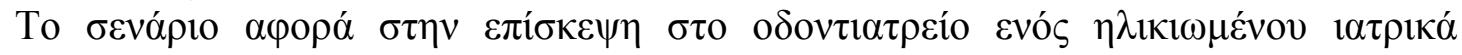

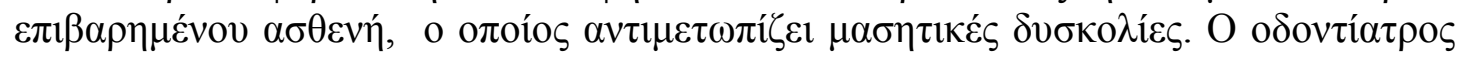

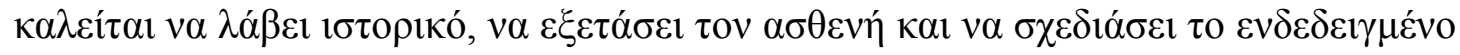

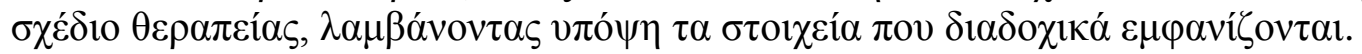

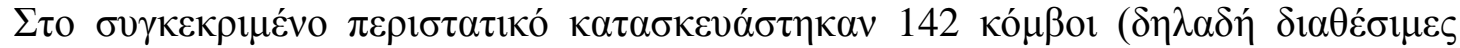

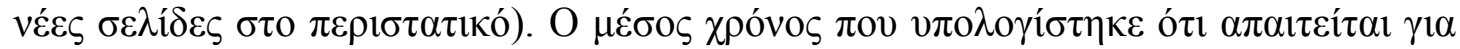

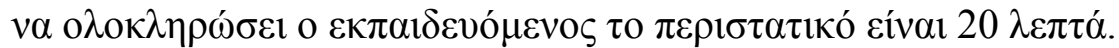

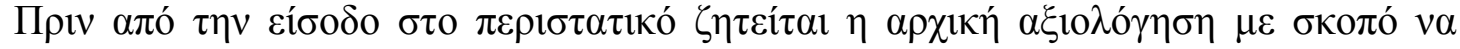

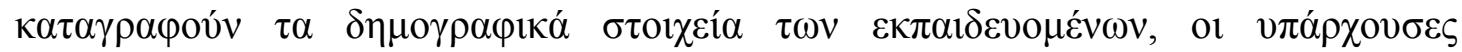

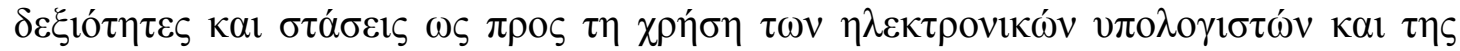

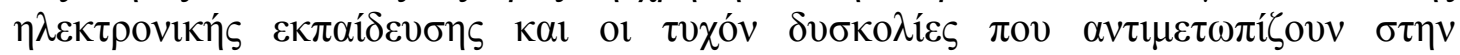

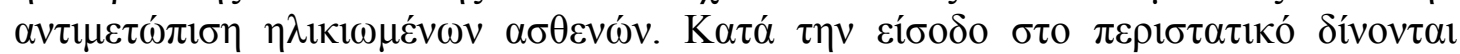

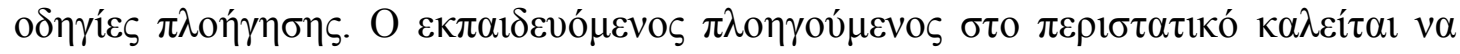

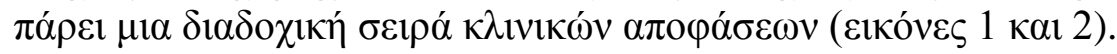

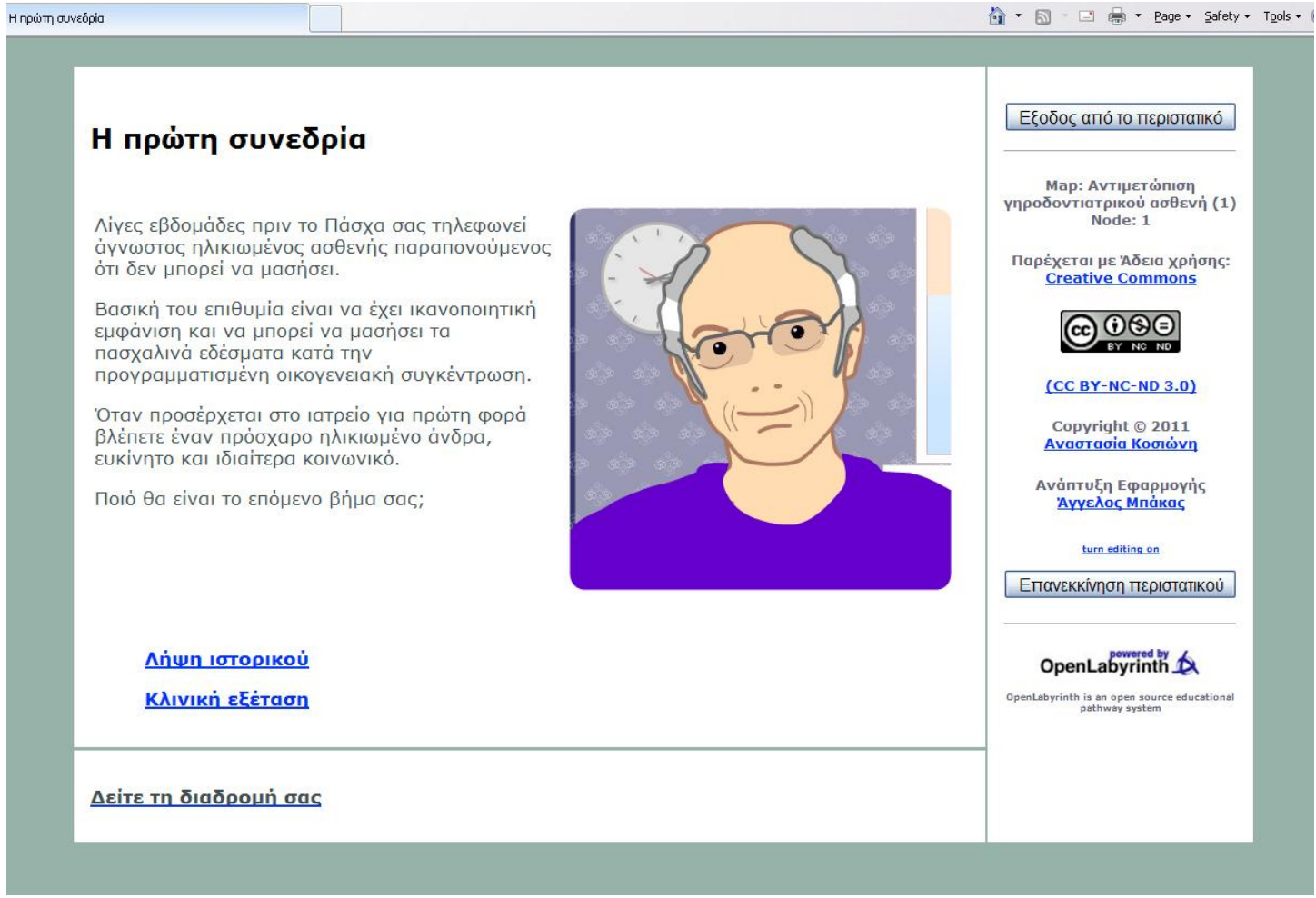

Eıкóva 1

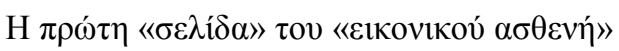




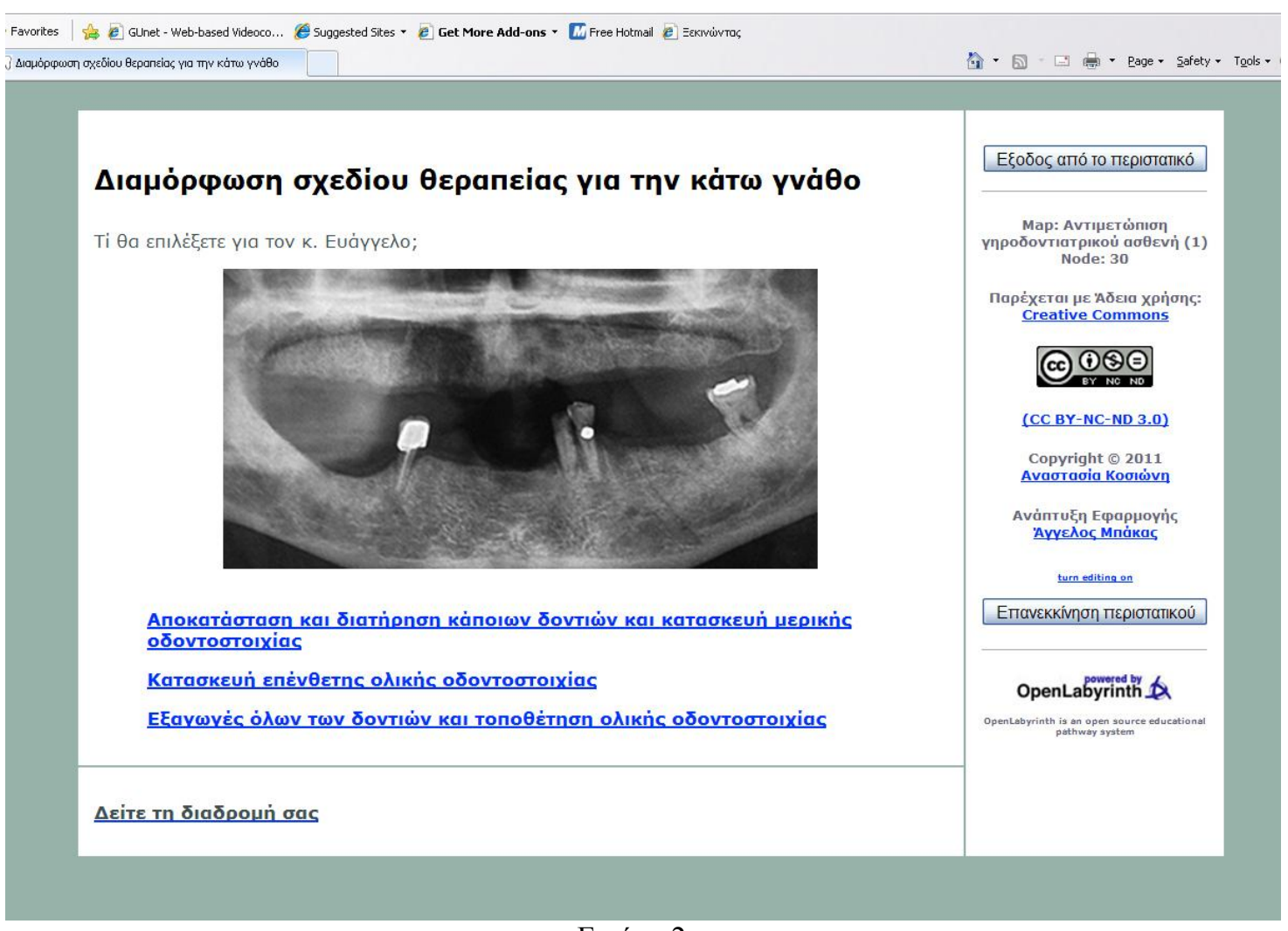

Eıкóva 2

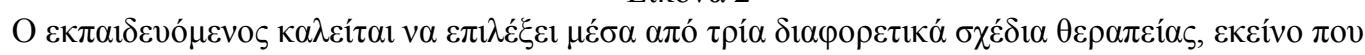

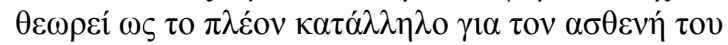

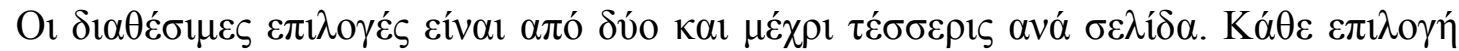

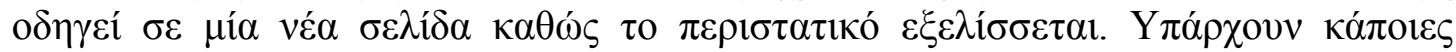

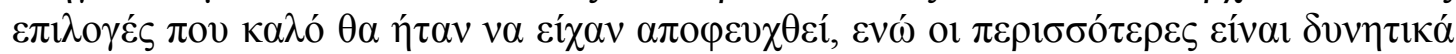

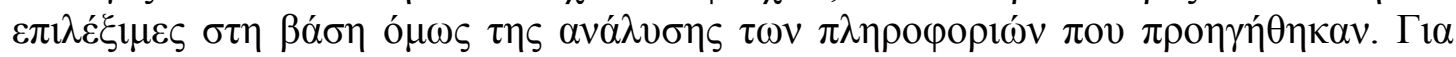

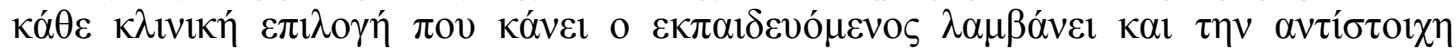

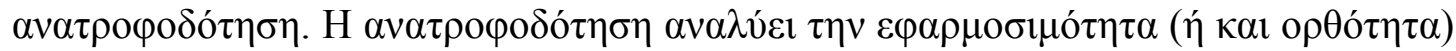

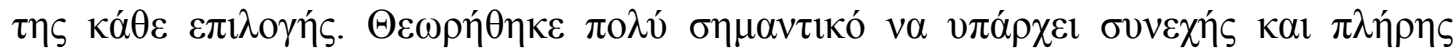

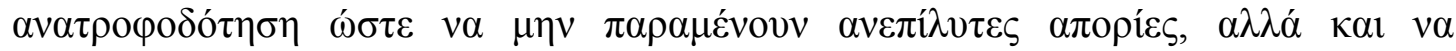

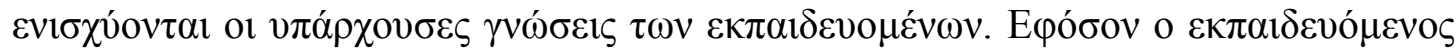

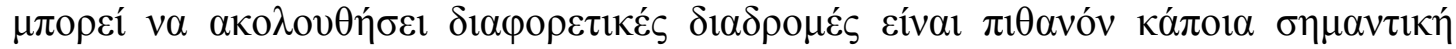

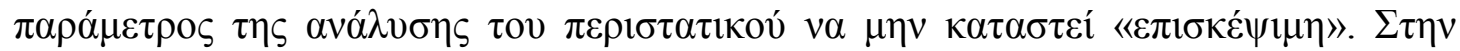

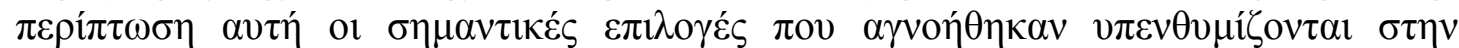

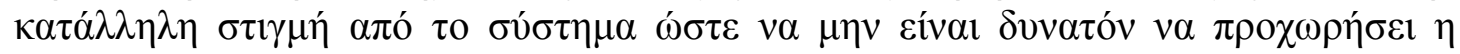

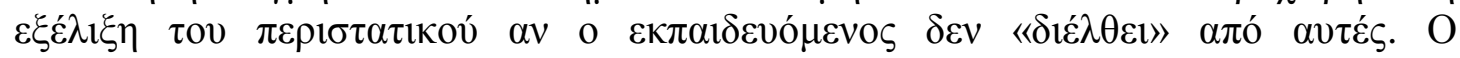

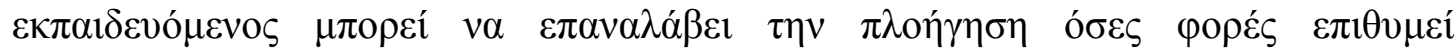

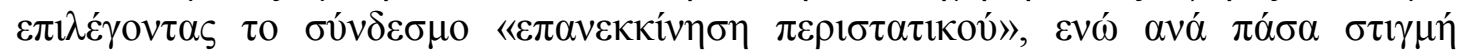

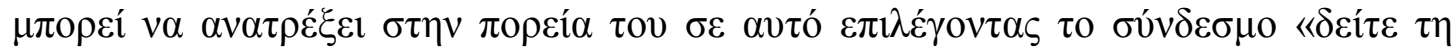

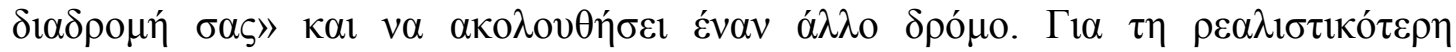

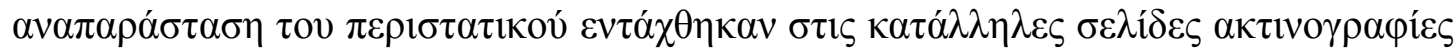

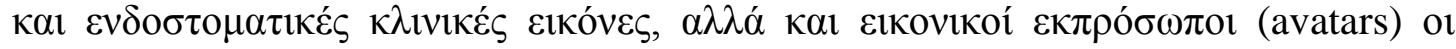

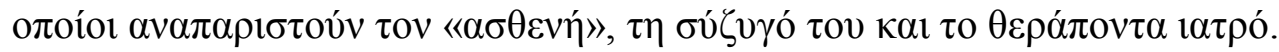




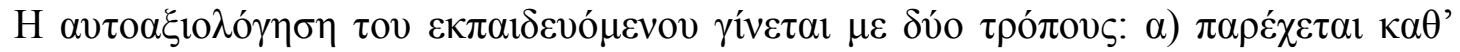

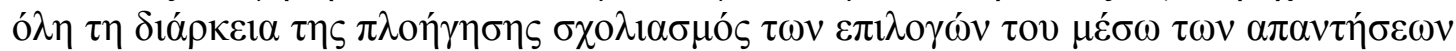

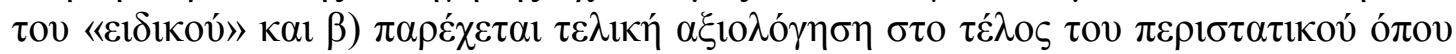

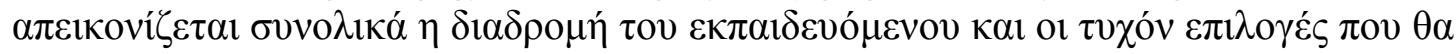

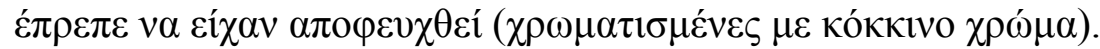

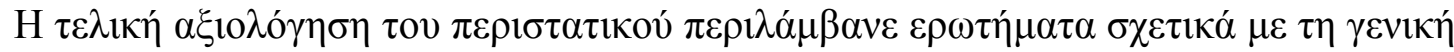

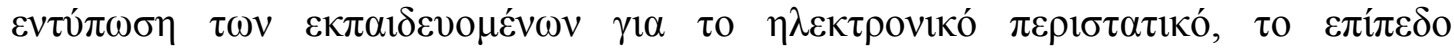

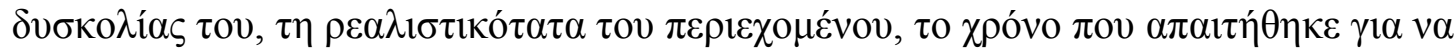

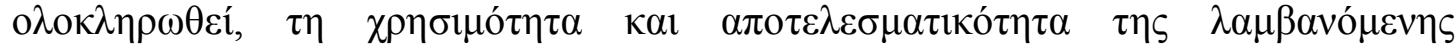

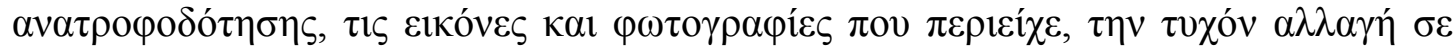

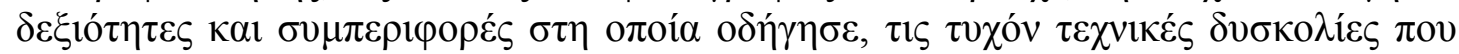

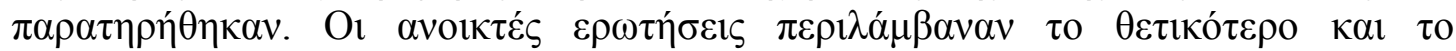

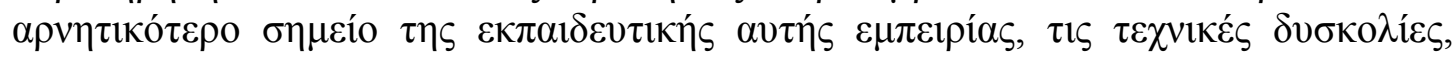

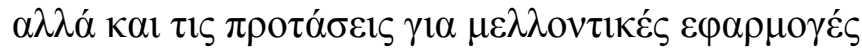

\section{$\Sigma v \zeta \dot{\eta} \tau \eta \sigma \eta$}

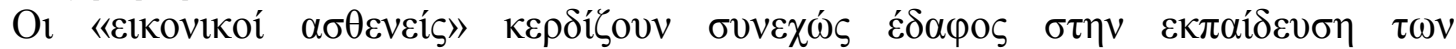

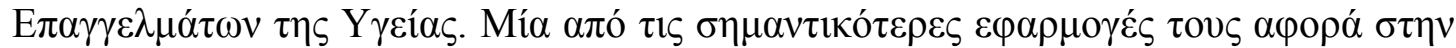

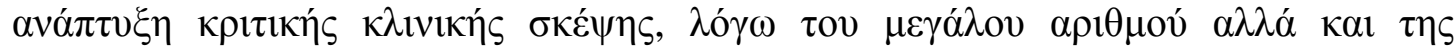

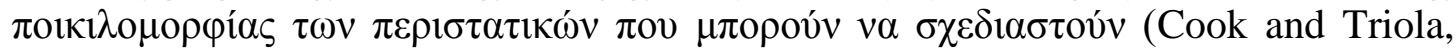

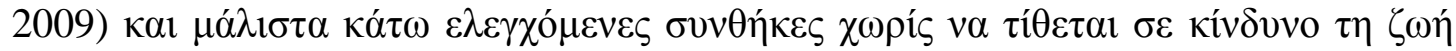

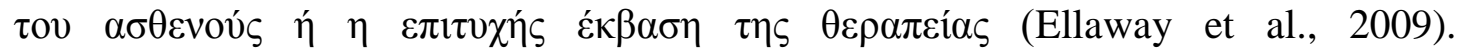

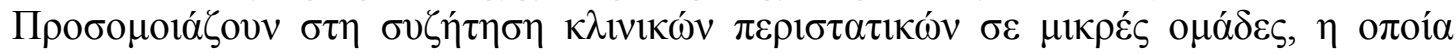

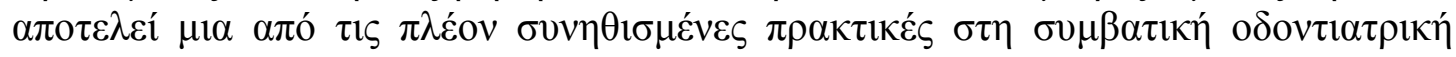

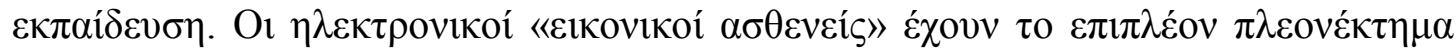

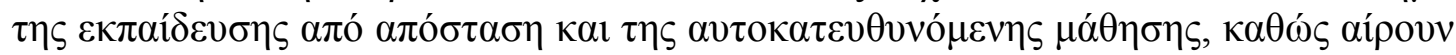

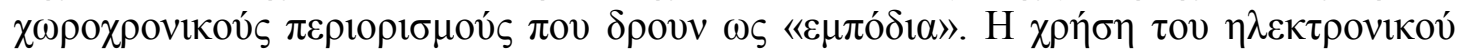

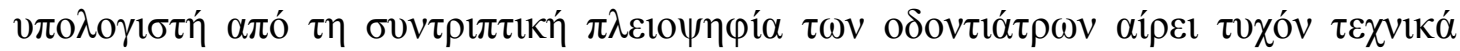

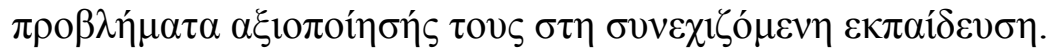

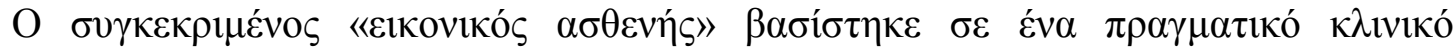

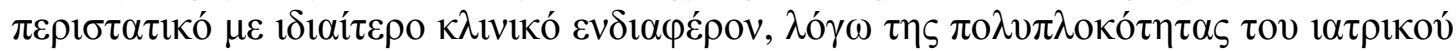

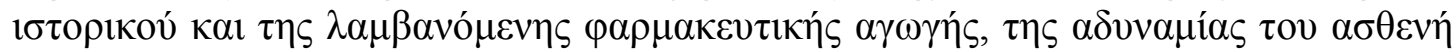

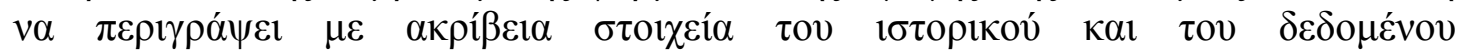

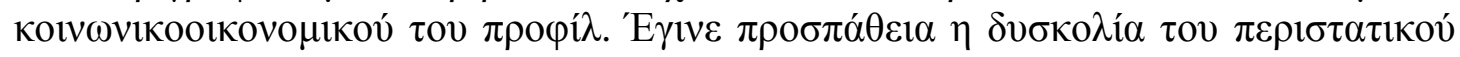

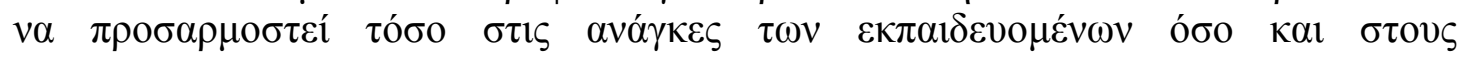

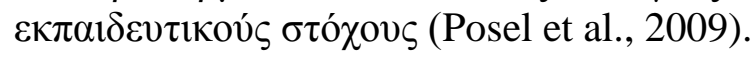

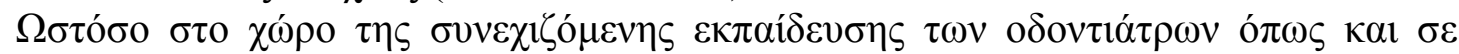

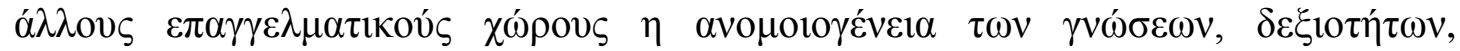

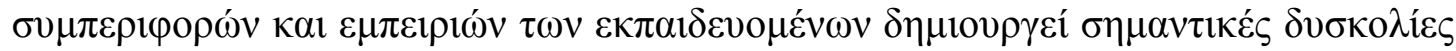

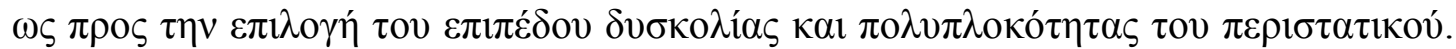

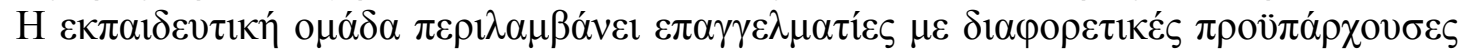

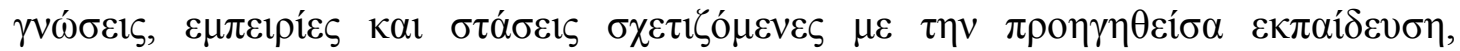

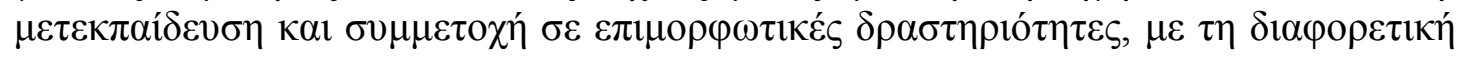

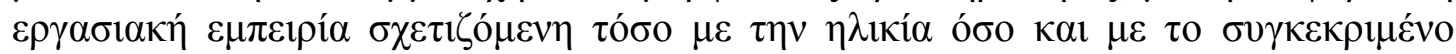

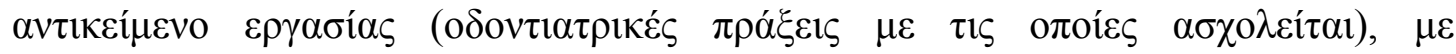

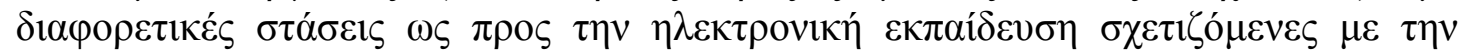

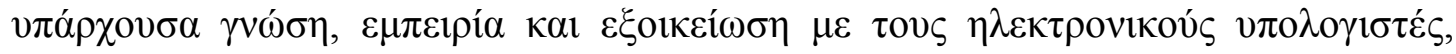




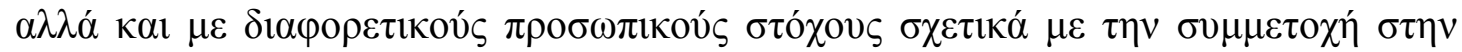
$\varepsilon \kappa \pi \alpha 1 \delta \varepsilon v \tau \imath \kappa \eta ́ ~ \delta 1 \alpha \delta 1 \kappa \alpha \sigma i ́ \alpha$.

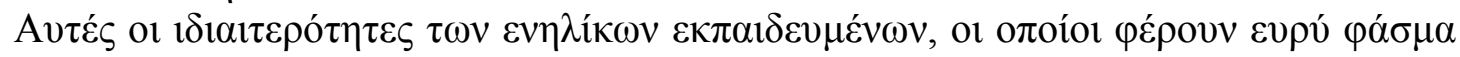

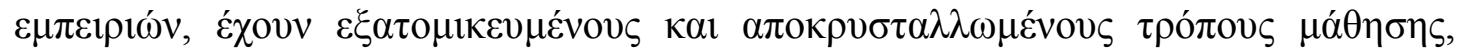

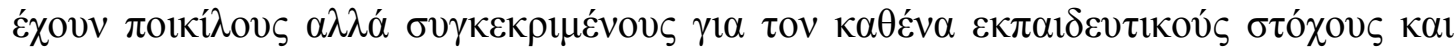

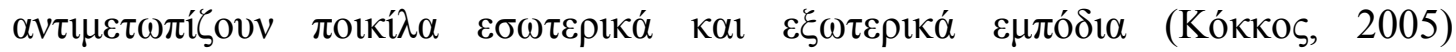

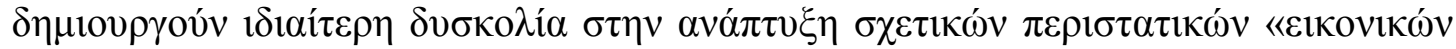
$\alpha \sigma \theta \varepsilon v \omega ́ v » \quad \gamma 1 \alpha \quad \tau \eta \quad \sigma v v \varepsilon \chi 1 \zeta o ́ \mu \varepsilon v \eta \quad \varepsilon \kappa \pi \alpha i \delta \varepsilon v \sigma \eta$. H $\tau \varepsilon \lambda 1 \kappa \eta \dot{~} \alpha \xi 10 \lambda o ́ \gamma \eta \sigma \eta \quad \tau \eta \varsigma$

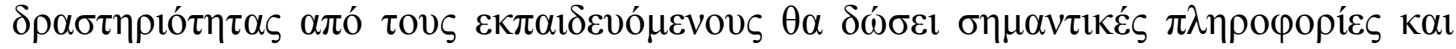

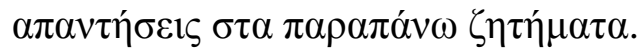

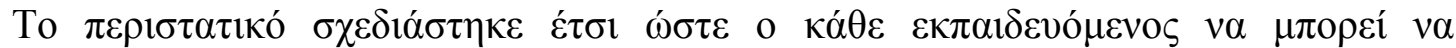

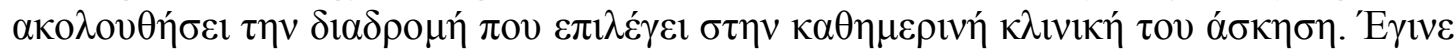

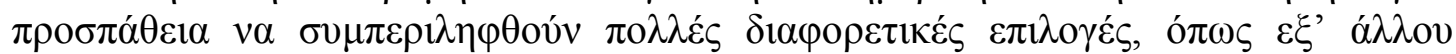

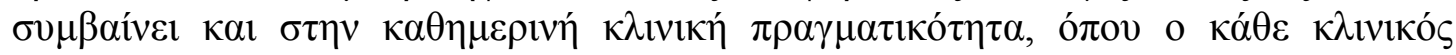

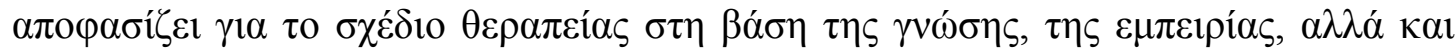

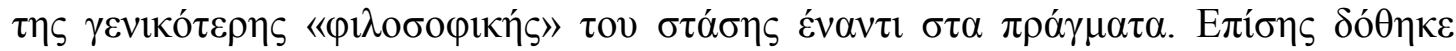

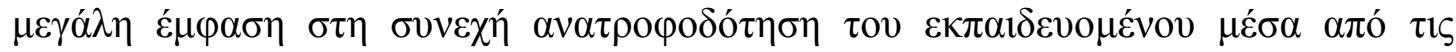

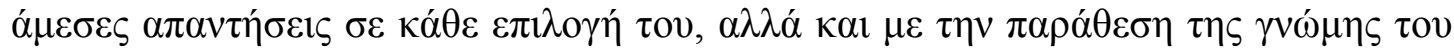

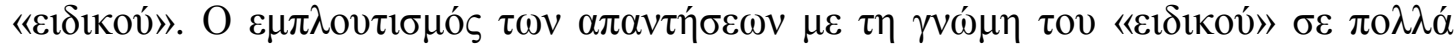

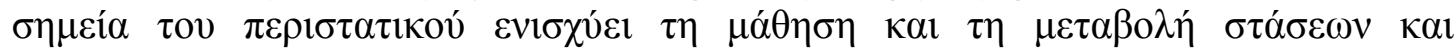

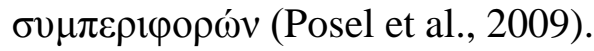

\section{$\Sigma v \mu \pi \varepsilon \rho \alpha ́ \sigma \mu \alpha \tau \alpha$}

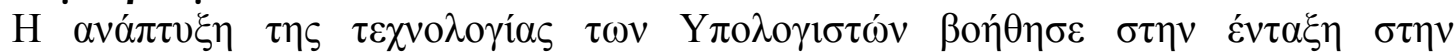

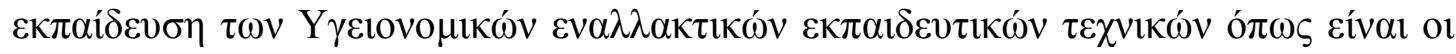

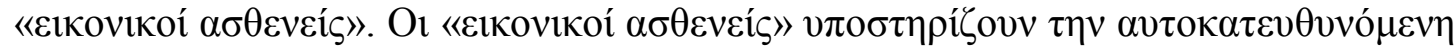

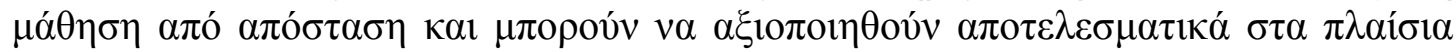

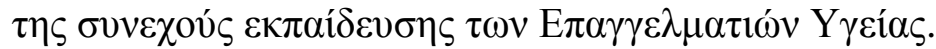

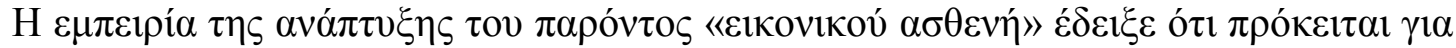

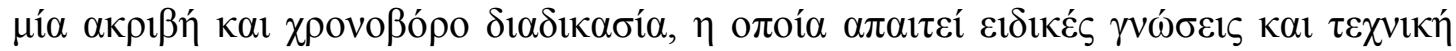

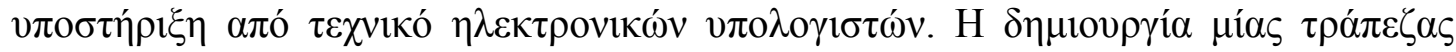

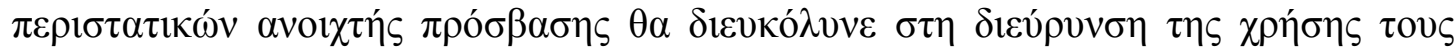

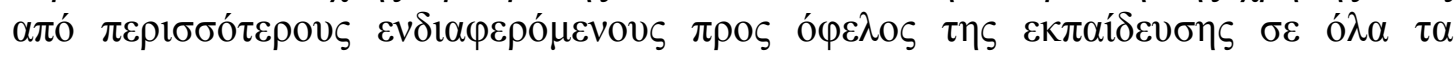
$\varepsilon \pi i ́ \pi \varepsilon \delta \alpha$.

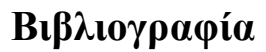

Ellaway R., Candler C., Greene P., Smothers V. (2006). An architectural model for MedBiquitous virtual patients. Retrieved on $30^{\text {th }}$ May 2011 from http://groups.medbiq.org/medbiq/display/VPWG/MedBiquitous+Virtual+Patient+Architecture

Ellaway R., Poulton T., Fors U., McGee JB., Albright S. (2008). Building a virtual patient commons. Medical Teacher, 30, pp. 170-174.

Ellaway RH., Poulton T., Smothers V., Greene P. (2009). Virtual patients come of age. Medical Teacher, 31, pp. 683-684.

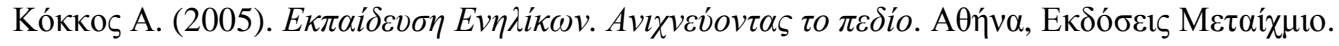

Kravitz AS., and Treasure ET. (2008). Manual of dental practice 2008 (version 4). The Council of European Dentists, pp. 31-39.

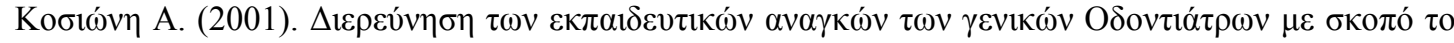

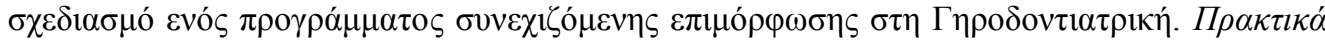

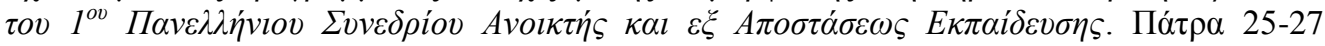

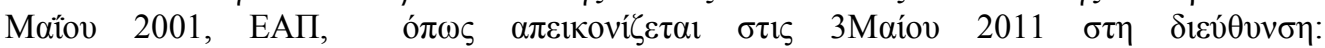
http://www.eap.gr/news/EXAGGELIA_SYNEDRIOU/synedrio/html/sect7/107.htm 


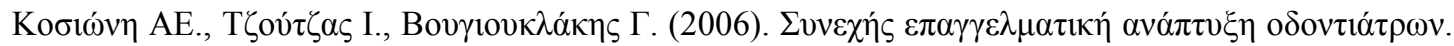

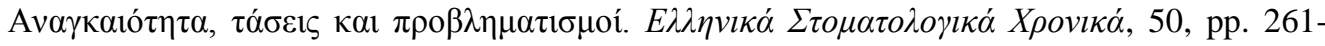
274.

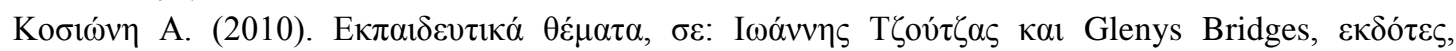

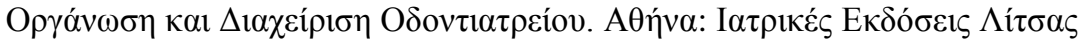

Cook DA., Triola MM. (2009). Virtual patients: a critical literature review and proposed next steps. Medical Education, 43, pp. 303-311.

Posel N., Fleiszer D., Shore BM. (2009). 12 tips: guidelines for authoring virtual patient cases. Medical Teacher, 31, pp. 701-708.

Hopcraft MS., Manton DJ., Chong PL., Ko G., Ong PYS., Sribalachandran S., Wang CJ., Yong PY., Jian LF. (2010). Participation in continuing professional development by dental practitioners in Victoria, Australia in 2007. European Journal of Dental Education, 14, pp. 227-234. 